\title{
Effects of Aerobic Exercise and Yoga Breathing Exercise (Pranayama) on Blood Pressure in Hypertensive Patients
}

\author{
Khairunissa1), Didik Gunawan Tamtomo²), Hanung Prasetya3) \\ 1)Masters Program in Public Health, Universitas Sebelas Maret \\ 2)Faculty of Medicine, Universitas Sebelas Maret \\ 3)Health Polytechnics, Ministry of Health Surakarta
}

\section{ABSTRACT}

Background: Uncontrolled hypertension or high blood pressure is a major risk factor for cardiovascular disease. Non-pharmacological treatment has become an important part of hypertension management. Aerobic exercise and yoga breathing exercise (pranayama) are types of non-pharmacological treatment that have an effect on lowering blood pressure. This study aims to examine the effect of aerobic exercise and yoga breathing exercise (pranayama) on blood pressure in hypertensive patients.

Subjects and Method: The meta-analysis was carried out according to the PRISMA guidelines. Articles with randomized controlled trial study designs published in 2010-2020 were included using databases from PubMed, Science Direct, Research Gate, and Google Scholer. Based on the database, there were 20 articles that met the inclusion criteria. This study involved 1564 subjects which were divided into, 953 aerobic exercise subjects and 611 yoga breathing exercise (pranayama) subjects. The analysis was carried out using RevMan 5.4 software.

Results: A total of 20 articles were reviewed in the meta-analysis. A total of 11 articles in the meta-analysis showed a statistically significant effect of the aerobic exercise intervention on reducing systolic blood pressure $(\mathrm{SMD}=-0.93$; 95\% $\mathrm{CI}=-1.33$ to $-0.53 ; \mathrm{p}<0.001)$ and diastolic blood pressure $(\mathrm{SMD}=-0.56 ; 95 \% \mathrm{CI}=-0.92$ to $-0.20 ; p=0.002)$. A total of 9 articles conducted meta-analysis showed a statistically significant effect of yoga breathing exercise (pranayama) intervention on reducing systolic blood pressure $(\mathrm{SMD}=-1.68 ; 95 \% \mathrm{CI}=-2.52$ to -0.84 ; $\mathrm{p}<0.001)$. There was a statistically insignificant effect on the decrease in diastolic blood pressure $(\mathrm{SMD}=-0.65 ; 95 \% \mathrm{CI}=-1.54$ to 0.25 ; $\mathrm{p}=0.16)$.

Conclusion: Aerobic exercise and yoga breathing exercise (pranayama) can lower blood pressure in hypertensive patients.

Keywords: Aerobic exercise, yoga breathing exercise, pranayama, blood pressure, hypertension, meta analysis

\section{Correspondence:}

Khairunissa. Masters Program in Public Health, Universitas Sebelas Maret. Jl. Ir. Sutami 36A, Surakarta 57126, Central Java. Email: khairunissahabibio2@gmail.com Mobile: 081779226622.

Cite this as:

Khairunissa, Tamtomo DG, Prasetya H (2021). Effects of Aerobic Exercise and Yoga Breathing Exercise (Pranayama) on Blood Pressure in Hypertensive Patients. J Epidemiol Public Health. 06(01): 134-147. https://doi.org/10.26911/jepublichealth.2021.06.02.01.

cc (i) (8) Journal of Maternal and Child Health is licensed under a Creative Commons EY NG SA Attribution-NonCommercial-ShareAlike 4.0 International License.

\section{BACKGROUND}

Hypertension or high blood pressure is a global health problem that affects nearly 1 billion people worldwide and is expected to increase to 1.5 billion by 2025 ( $\mathrm{Fu}$ et al.,
2020). Hypertension is the leading cause of death globally accounting for 10.4 million deaths each year (Unger et al., 2020).

Uncontrolled hypertension is a major risk factor for cardiovascular diseases such 
as Coronary Artery Disease (CAD), stroke, Heart Failure (HF) and Chronic Kidney Disease (CKD) (Weber et al., 2014). Dorobantu et al., (2019) stated that hypertension was the highest cause in the population with heart failure compared to other causes of $82-91 \%$. In addition, someone with uncontrolled hypertension has a risk of having a stroke 7 times compared to those who control their hypertension, 6 times for experiencing heart failure, and three times for having a heart attack (Lindasari et al., 2020).

As a form of control for hypertension, there are two kinds of interventions that can be done, namely pharmacological and non-pharmacological (Astuti et al., 2016). Even if pharmacological treatment or taking antihypertensive drugs is very effective, about $70 \%$ of patients who only receive antihypertensive drugs, their blood pressure cannot be controlled. This condition encourages non-pharmacological interventions to play a role in preventing and controlling hypertension. Non-pharmacological hypertension interventions can be used alone before starting pharmacological therapy or in combination with pharmacology (Mahmood et al., 2018).

The American, Canadian, and European hypertension guidelines recommend non-pharmacological interventions in the prevention and management of hypertension (Williams et al., 2018) (Whelton et al., 2018) (Leung et al., 2017). Nonpharmacological interventions help reduce the daily dose of antihypertensive drugs and delay the progression from prehypertension to the hypertensive stage. These interventions include lifestyle modification, improving diet or diet, physical exercise or sports, efforts to reduce stress, and minimizing alcohol consumption (Mahmood et al., 2018).
Aerobic exercise and yoga breathing exercise (pranayama) are non-pharmacological treatments recommended by European and American hypertension guidelines to lower blood pressure in hypertensive patients. (WHO, 2013).

In general, aerobics is useful in increasing fitness (Armstrong et al., 2017), while yoga is useful in reducing stress levels or emotions and anxiety, especially for those who are in certain conditions such as illness or pregnancy (Aflahiyah et al., 2020; Ningrum et al. al., 2019).

In a study by Daimo et al., (2020) entitled "Effect of aerobic exercise on blood pressure in men with hypertension: A randomized controlled study," the administration of aerobic exercise can significantly reduce systolic blood pressure $(\mathrm{p}=0.001)$ and diastolic blood pressure $(\mathrm{p}=0.001) . \mathrm{p}=$ o.001). In addition, in a study by Dixit et al., (2017) entitled "An evaluation of the effect of Pranayamas and Transcendental meditation on high blood pressure subjects: A non-blinded randomized controlled trial," the administration of pranayama was assessed to reduce systolic and diastolic blood pressure. significantly $(\mathrm{p}<$.001).

This study is expected to prove the effect of giving aerobic exercise and yoga breathing exercise (pranayama) to lower blood pressure in hypertensive patients.

\section{SUBJECTS AND METHOD}

\section{Study Design}

This was a systematic review and meta-analysis with PRISMA flow diagram guidelines. Search articles using databases: PubMed, Science Direct, Research Gate, and Google Scholars. Keywords used for the first intervention: "aerobic exercise" OR "aerobic training" AND "blood pressure" AND hypertension OR hypertensive OR "patients with hypertension" AND "randomized controlled trial". And keywords for the second 
intervention: pranayama OR "pranayama breathing" OR "yoga breathing" AND "blood pressure" AND hypertension OR hypertensive OR "patients with hypertension" AND "randomized controlled trial".

\section{Inclusion Criteria}

The inclusion criteria for this research article are full paper randomized controlled trails (RCT) articles, using aerobic exercise and yoga breathing exercise (pranayama) interventions, the subject is patients with a diagnosis of hypertension and systolic and diastolic blood pressure outcomes.

\section{Exclusion Criteria}

The exclusion criteria for this research article are articles that do not use English, and articles published before 2010.

\section{Operational Definition of Variables}

Articles included in the study were PICOadjusted. The search for articles was carried out by considering the eligibility criteria defined using the PICO model. There are two PICO in this study, first, the research population, namely hypertensive patients, using aerobic exercise intervention and non-aerobic exercise control and blood pressure outcomes. The second PICO, the research population, namely hypertensive patients, used intervention yoga breathing exercise (pranayama) and control non-yoga breathing exercise (pranayama) as well as blood pressure outcomes.

Aerobic exercise is physical exercise that involves the large muscles of the body rhythmically and continuously, requiring an aerobic metabolic pathway as a source of energy supply with a frequency of exercise 3-7 days/week, moderate intensity 50\%$80 \%$, duration of exercise 30-300 minutes/ week, sessions in the form of brisk walking, jogging, cycling, gymnastics, and swimming.

\section{Yoga breathing exercise (pranayama)}

is a breathing exercise in yoga that is done slowly and deeply which has several kinds of techniques such as sheetali pranayama, bhramari pranayama, ujjayi pranayama, bhasrika pranayama, Kapalbhati pranayama, and anuloma viloma pranayama.

Blood pressure is a number that describes the highest and lowest pressures in the blood vessels when the heart contracts and when it relaxes, consisting of an upper or systolic number and a lower or diastolic number with units of millimetres of mercury (mmHg).

\section{Study Instruments}

The study was conducted using the PRISMA flow diagram guidelines and the assessment of the quality of research articles using the Critical Appraisal Checklist for RCT Study tools (CEBM, 2014).

\section{Data Analysis}

The data in this study were analyzed using the RevMan 5.3 application, to calculate the effect size and heterogeneity of the study. The results of data processing are presented in the form of forest plots and funnel plots.

\section{RESULTS}

The article review process was carried out using the PRISMA flow chart, which can be seen in Figure 1. The total articles obtained were 20 articles. The distribution of the article is on 4 continents with details 9 from Asia, 5 from Europe, 3 from Africa, and 3 from America.

\section{The effect of aerobic exercise on blood pressure in hypertensive patients}

Table 1 shows 11 RCT articles on the effect of aerobic exercise on blood pressure in hy pertensive patients who meet qualitative and quantitative requirements.

\section{a. Forest plot}

Figure 2 shows that there is a statistically significant effect of the aerobic exercise intervention on reducing systolic and diastolic blood pressure. Giving aerobic exercise to hypertensive patients was able to reduce 
Khairunissa et al./ Aerobic Exercise and Yoga Breathing Exercise (Pranayama) on Blood Pressure

systolic blood pressure 0.93 units lower than non-aerobic exercise interventions $(\mathrm{SMD}=-0.93 ; 95 \% \mathrm{CI}=-1.33$ to $-0.53 ; \mathrm{p}<$ 0.001).

Figure 3 shows that there is a statistically significant effect of the aerobic exercise intervention on reducing diastolic blood pressure. Giving aerobic exercise to hypertensive patients can reduce diastolic blood pressure 0.56 units lower than non-aerobic exercise interventions $(\mathrm{SMD}=-0.56 ; 95 \%$ $\mathrm{CI}=-0.92$ to $-0.20 ; \mathrm{p}=0.002$ ).

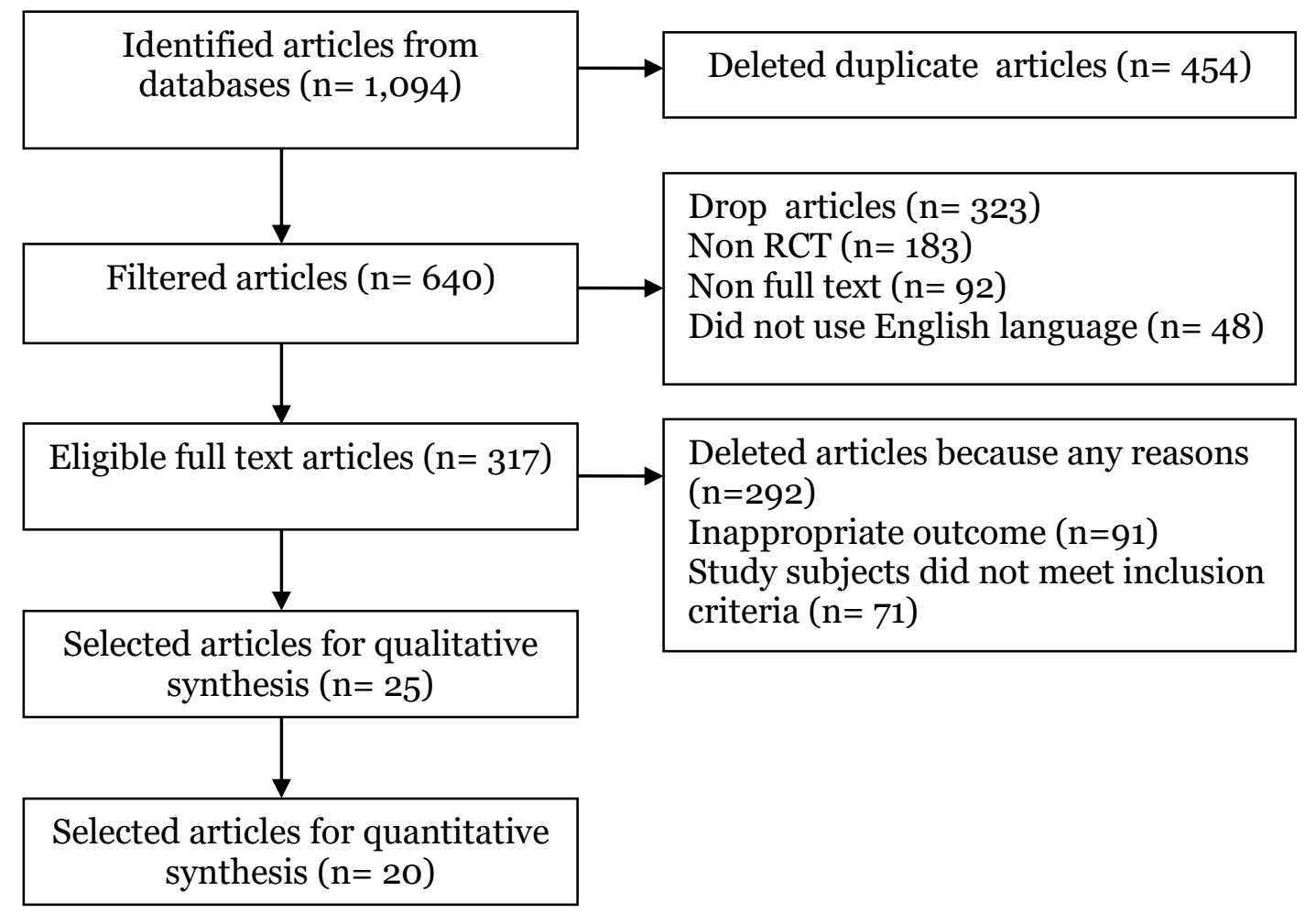

Figure 1. PRISMA flow chart

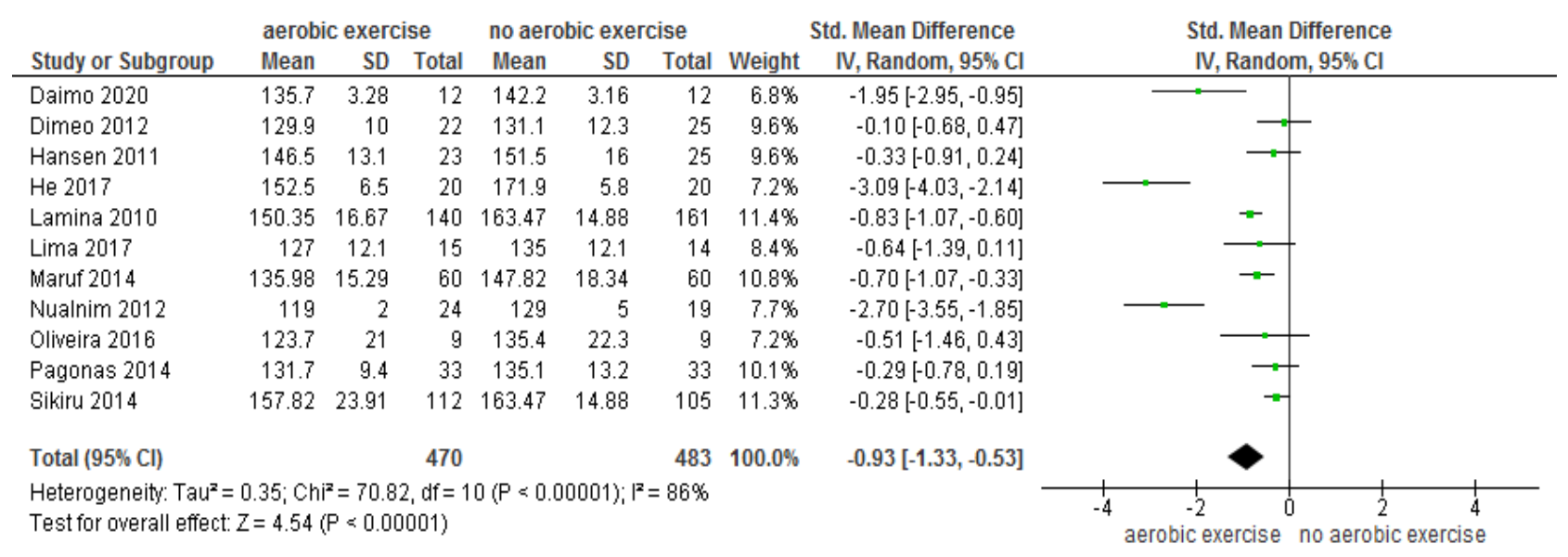

Figure 2. Forest plot of the effect of aerobic exercise on systolic blood pressure in hypertensive patients 
Khairunissa et al./ Aerobic Exercise and Yoga Breathing Exercise (Pranayama) on Blood Pressure

Table 1. Effect of aerobic exercise on blood pressure in hypertensive patients

\begin{tabular}{|c|c|c|c|c|c|}
\hline $\begin{array}{l}\text { Author } \\
\text { (Year) }\end{array}$ & Country & Population & Intervention & Comparison & Outcome \\
\hline $\begin{array}{l}\text { Daimo } \\
(2020)\end{array}$ & Ethiopia & $\begin{array}{l}\text { Grade } 1 \text { hyperten- } \\
\text { sion patient, male } \\
\text { aged } 31-45 \text { years }\end{array}$ & $\begin{array}{l}\text { Brisk walk } 45 \\
\text { minutes/ session, } 3 \\
\text { times a week for } 16 \\
\text { weeks, moderate } \\
\text { intensity (60\% HR } \\
\text { max) }\end{array}$ & $\begin{array}{l}\text { No practice at } \\
\text { all }\end{array}$ & $\begin{array}{l}\text { Systolic and } \\
\text { diastolic blood } \\
\text { pressure }\end{array}$ \\
\hline $\begin{array}{l}\text { Dimeo } \\
(2012)\end{array}$ & German & $\begin{array}{l}\text { Grade } 1 \text { hyperten- } \\
\text { sive patients, male } \\
\text { and female }\end{array}$ & $\begin{array}{l}\text { Brisk walking on the } \\
\text { treadmill } 3 \text { times a } \\
\text { week, for } 12 \text { weeks }\end{array}$ & $\begin{array}{l}\text { No practice at } \\
\text { all }\end{array}$ & $\begin{array}{l}\text { Systolic and } \\
\text { diastolic blood } \\
\text { pressure }\end{array}$ \\
\hline $\begin{array}{l}\text { Hansen } \\
\text { (2011) }\end{array}$ & Norway & $\begin{array}{l}\text { Grade } 1 \text { and } 2 \\
\text { essential hyper- } \\
\text { tension patients, } \\
\text { women < } 65 \text { years } \\
\text { old }\end{array}$ & $\begin{array}{l}\text { Brisk walking on a } \\
\text { treadmill } 47 \\
\text { minutes/session, for } \\
12 \text { weeks, moderate } \\
\text { intensity ( } 70 \% \mathrm{HR} \\
\text { max) }\end{array}$ & $\begin{array}{l}\text { No practice at } \\
\text { all }\end{array}$ & $\begin{array}{l}\text { Systolic blood } \\
\text { pressure, } \\
\text { diastolic, heart } \\
\text { rate, quality of } \\
\text { life (QoL) }\end{array}$ \\
\hline $\begin{array}{l}\mathrm{He} \\
(2017)\end{array}$ & China & $\begin{array}{l}\text { Patients with grade } \\
1 \text { hypertension, men } \\
\text { and women aged } \\
55-60 \text { years }\end{array}$ & $\begin{array}{l}\text { Brisk walking } 30 \\
\text { minutes/session, for } \\
12 \text { weeks, moderate } \\
\text { intensity }\end{array}$ & $\begin{array}{l}\text { No practice at } \\
\text { all }\end{array}$ & $\begin{array}{l}\text { Systolic and } \\
\text { diastolic blood } \\
\text { pressure }\end{array}$ \\
\hline $\begin{array}{l}\text { Lamina } \\
(2010)\end{array}$ & Nigeria & $\begin{array}{l}\text { Patients with grade } \\
1 \text { and } 2 \text { hyperten- } \\
\text { sion, men and } \\
\text { women aged 50-70 } \\
\text { years }\end{array}$ & (50\% HR max) & $\begin{array}{l}\text { No practice at } \\
\text { all }\end{array}$ & $\begin{array}{l}\text { Systolic blood } \\
\text { pressure, } \\
\text { diastolic, heart } \\
\text { rate, VO2 max }\end{array}$ \\
\hline $\begin{array}{l}\text { Lima } \\
(2017)\end{array}$ & Brazil & $\begin{array}{l}\text { Stage } 2 \text { hyperten- } \\
\text { sion patients, men } \\
\text { and women aged } \\
60-75 \text { years }\end{array}$ & $\begin{array}{l}\text { Cycling } 45 \\
\text { minutes/session, for } \\
8 \text { weeks, moderate } \\
\text { intensity }\end{array}$ & $\begin{array}{l}\text { No practice at } \\
\text { all }\end{array}$ & $\begin{array}{l}\text { Systolic and } \\
\text { diastolic blood } \\
\text { pressure }\end{array}$ \\
\hline $\begin{array}{l}\text { Maruf } \\
(2014)\end{array}$ & Nigeria & $\begin{array}{l}\text { Essential hyperten- } \\
\text { sion patients, men } \\
\text { and women aged } 65 \\
\text { years }\end{array}$ & (6o-79\% HR max) & $\begin{array}{l}\text { No practice at } \\
\text { all }\end{array}$ & $\begin{array}{l}\text { Systolic and } \\
\text { diastolic blood } \\
\text { pressure }\end{array}$ \\
\hline $\begin{array}{l}\text { Nualnim } \\
\text { (2012) }\end{array}$ & Texas & $\begin{array}{l}\text { Patients with grade } \\
1 \text { hypertension and } \\
\text { prehypertension, } \\
\text { men and women } \\
\text { aged 50-80 years }\end{array}$ & $\begin{array}{l}\text { Aerobic exercise } 30 \\
\text { minutes/session for } \\
10 \text { weeks }\end{array}$ & $\begin{array}{l}\text { No practice at } \\
\text { all }\end{array}$ & $\begin{array}{l}\text { Systolic and } \\
\text { diastolic blood } \\
\text { pressure }\end{array}$ \\
\hline $\begin{array}{l}\text { Oliveria } \\
(2016)\end{array}$ & Portugal & $\begin{array}{l}\text { Grade } 1 \text { and } 2 \\
\text { essential hyperten- } \\
\text { sion patients, men } \\
\text { and women aged } \\
\text { 80-90 years }\end{array}$ & $\begin{array}{l}\text { Aerobic dance } 45 \\
\text { minutes/session, } 3 \\
\text { times a week, for } 12 \\
\text { weeks, moderate } \\
\text { intensity }\end{array}$ & $\begin{array}{l}\text { No practice at } \\
\text { all }\end{array}$ & $\begin{array}{l}\text { Systolic blood } \\
\text { pressure, } \\
\text { diastolic, heart } \\
\text { rate }\end{array}$ \\
\hline $\begin{array}{l}\text { Pagonas } \\
(2014)\end{array}$ & German & $\begin{array}{l}\text { Patients with grade } \\
1 \text { and } 2 \text { hyperten- } \\
\text { sion, men and } \\
\text { women aged } 60 \\
\text { years }\end{array}$ & (50-70\% HR max) & $\begin{array}{l}\text { No practice at } \\
\text { all }\end{array}$ & $\begin{array}{l}\text { Systolic and } \\
\text { diastolic blood } \\
\text { pressure }\end{array}$ \\
\hline $\begin{array}{l}\text { Sikiru } \\
(2014)\end{array}$ & Nigeria & $\begin{array}{l}\text { Grade } 1 \text { and } 2 \\
\text { essential hyperten- } \\
\text { sion patients, men } \\
\text { aged } 50-70 \text { years }\end{array}$ & $\begin{array}{l}\text { Swim } 45 \\
\text { minutes/session for } \\
12 \text { weeks, moderate } \\
\text { intensity }\end{array}$ & $\begin{array}{l}\text { No practice at } \\
\text { all }\end{array}$ & $\begin{array}{l}\text { Systolic blood } \\
\text { pressure, } \\
\text { diastolic, VO2 } \\
\text { max }\end{array}$ \\
\hline
\end{tabular}


Khairunissa et al./ Aerobic Exercise and Yoga Breathing Exercise (Pranayama) on Blood Pressure

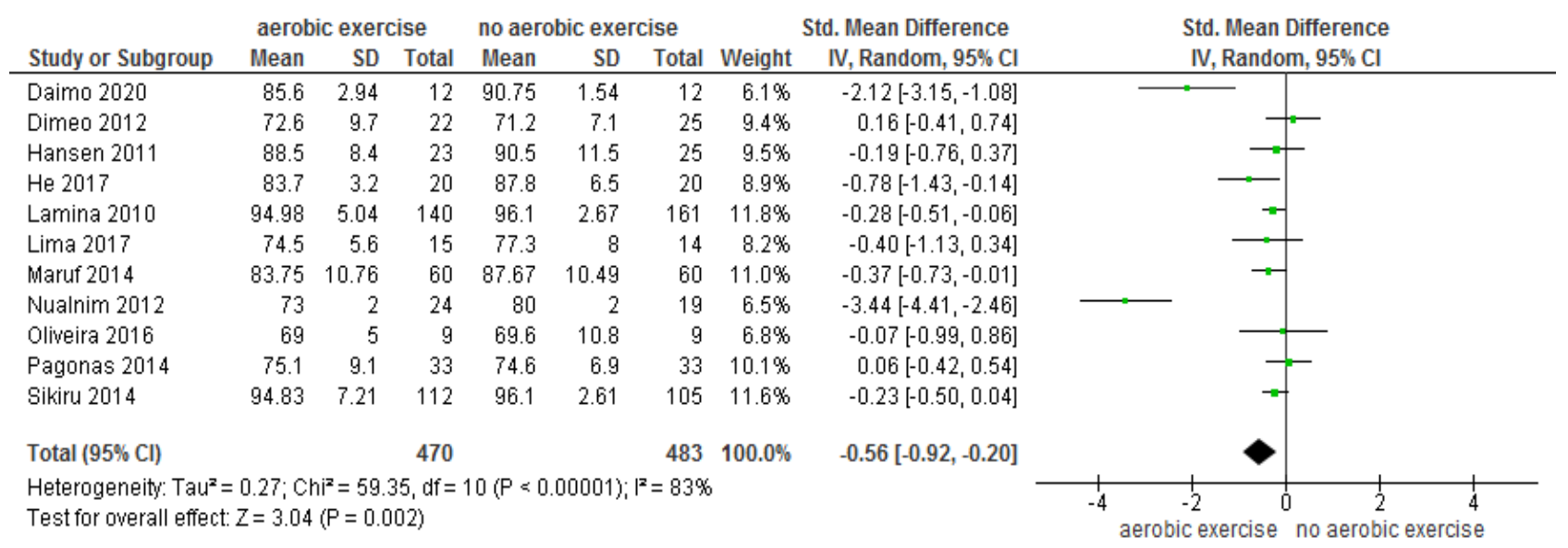

\section{Figure 3. Forest plot of the effect of aerobic exercise on diastolic blood pressure in hypertensive patients}

\section{b. Funnel plot}

In Figure 4, the funnel plot graph shows an asymmetrical distribution of the primary studies, 3 plots on the left and 8 plots on the right which indicates there is little publication bias and tends to overestimate the true effect of aerobic exercise intervention on lowering diastolic blood pressure (overestimate).

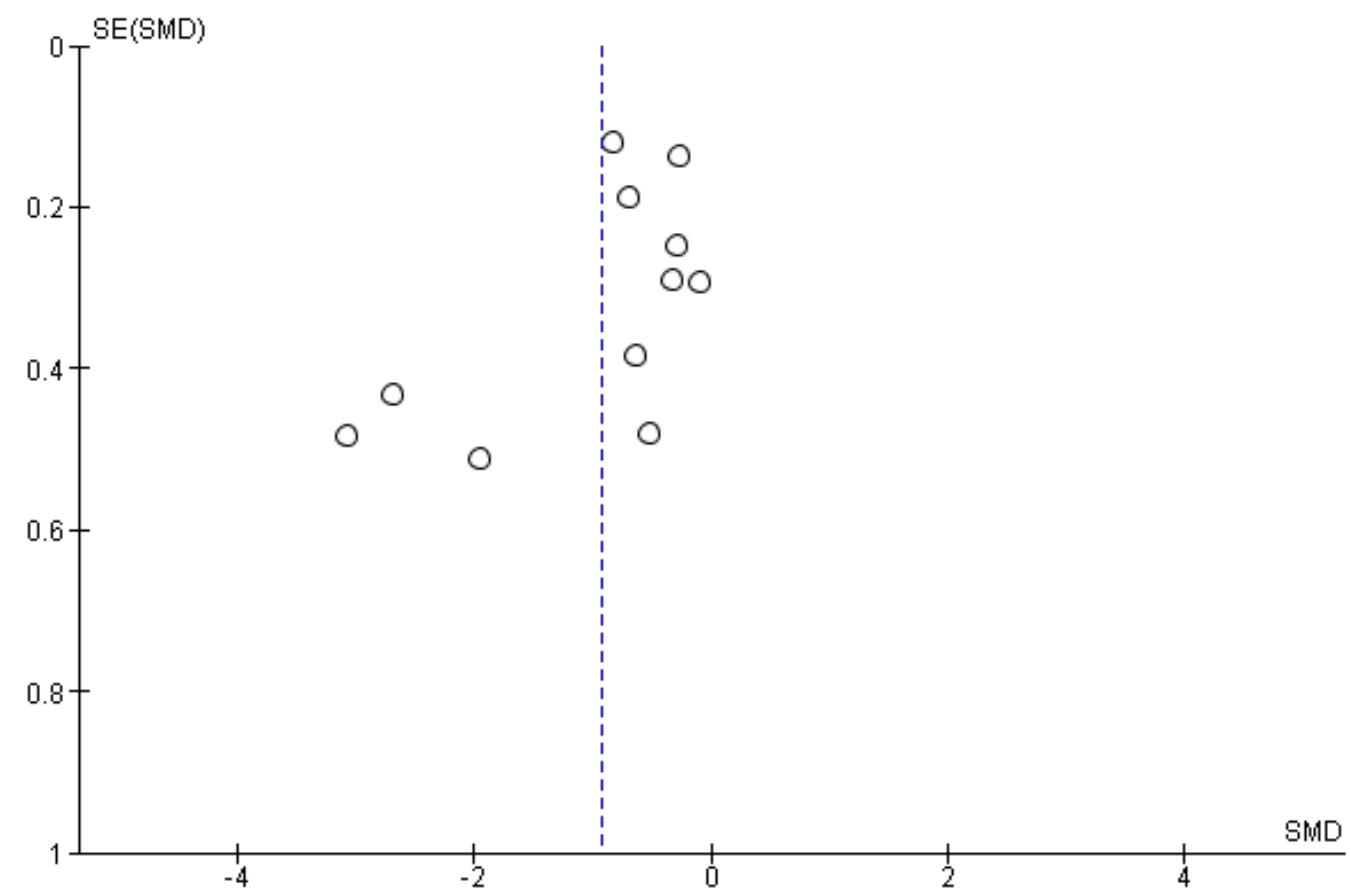

Figure 4. Funnel plot Effect of aerobic exercise on systolic blood pressure in hypertensive patients
In Figure 5, the funnel plot graph shows an asymmetrical distribution of the primary studies, 3 plots on the left and 8 plots on the right indicating there is a slight publiccation bias that tends to overestimate the true effect of aerobic exercise intervention on reducing diastolic blood pressure (overestimate). 


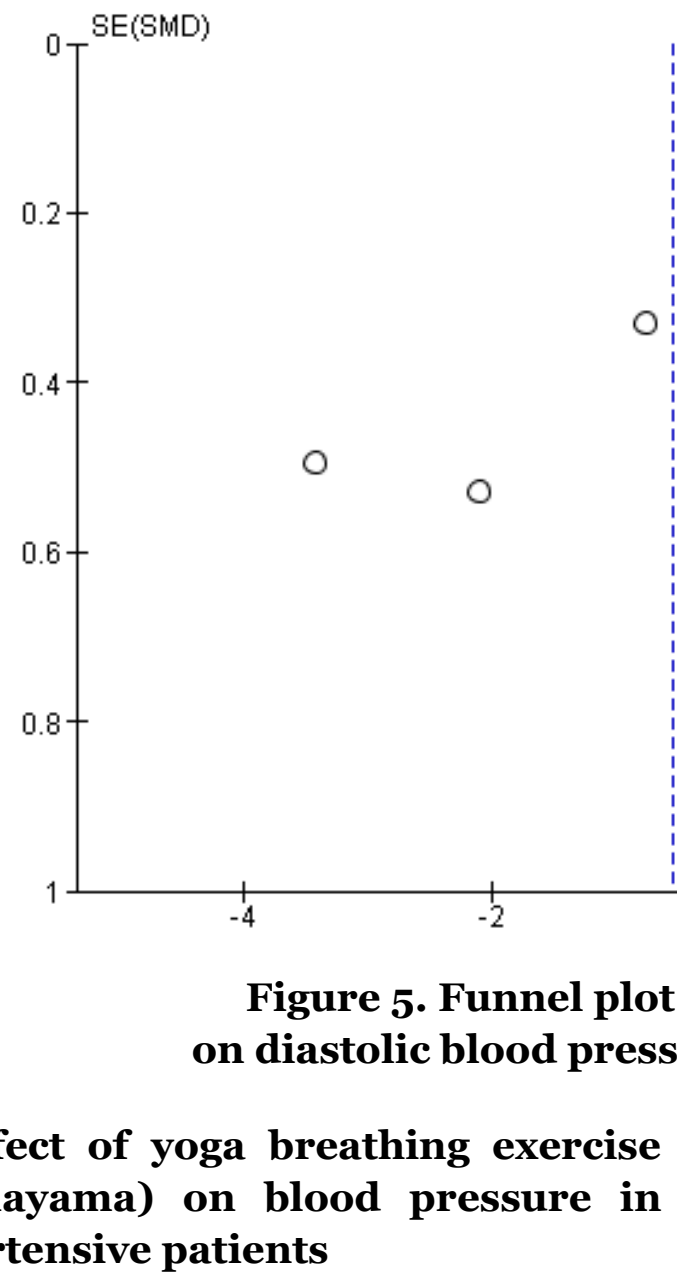

Table 2 shows 9 RCT articles on the effect of yoga breathing exercise (pranayama) on blood pressure in hypertensive patients who meet qualitative and quantitative requirements.

\section{a. Forest plot}

Figure 6 shows that there is a statistically significant effect of the yoga breathing exercise (pranayama) intervention on reducing systolic blood pressure. Giving yoga breathing exercise (pranayama) to hypertensive patients was able to reduce systolic blood pressure 1.68 units lower than non yoga breathing exercise (pranayama) intervention $(\mathrm{SMD}=-1.68 ; 95 \% \mathrm{CI}=-2.52$ to -0.84 ; $\mathrm{p}<0.001)$.

Figure 7 shows that there is a statistically insignificant effect of the yoga breathing exercise (pranayama) intervention on decreasing diastolic blood pressure. Giving yoga breathing exercise (pranayama) to hypertensive patients was able to reduce diastolic blood pressure 0.65 units lower than the non-yoga breathing exercise (pranayama) intervention $(\mathrm{SMD}=-0.65$; $95 \% \mathrm{CI}=-1.54$ to $0.25 ; \mathrm{p}=0.16$ ). 
Table 2. Effect of yoga breathing exercise (pranayama) on blood pressure in hypertensive patients

\begin{tabular}{|c|c|c|c|c|c|}
\hline $\begin{array}{c}\text { Authors } \\
\text { (year) }\end{array}$ & Country & Population & $\begin{array}{c}\text { Inter- } \\
\text { vention }\end{array}$ & Comparison & Outcome \\
\hline $\begin{array}{l}\text { Dixit } \\
(2017)\end{array}$ & India & $\begin{array}{l}\text { Hypertensive } \\
\text { patients, men and } \\
\text { women aged 40-55 } \\
\text { years }\end{array}$ & $\begin{array}{l}\text { Pranayama } \\
\text { for } 12 \text { weeks }\end{array}$ & $\begin{array}{l}\text { No practice at } \\
\text { all }\end{array}$ & $\begin{array}{l}\text { Systolic blood } \\
\text { pressure, dia- } \\
\text { stolic, heart } \\
\text { rate, respire- } \\
\text { tory rate }\end{array}$ \\
\hline $\begin{array}{l}\text { Ghati } \\
(2020)\end{array}$ & India & $\begin{array}{l}\text { Patients with } \\
\text { hypertension stage } 1 \\
\text { and } 2 \text {, men and } \\
\text { women aged 30-70 } \\
\text { years }\end{array}$ & $\begin{array}{l}\text { Bee } \\
\text { Humming } \\
\text { Breathing } 5 \\
\text { minutes/ } \\
\text { session }\end{array}$ & $\begin{array}{l}\text { No practice at } \\
\text { all }\end{array}$ & $\begin{array}{l}\text { Systolic blood } \\
\text { pressure, } \\
\text { diastolic, heart } \\
\text { rate, }\end{array}$ \\
\hline $\begin{array}{l}\text { Goyal } \\
(2016)\end{array}$ & India & $\begin{array}{l}\text { Patients with grade } 1 \\
\text { hypertension, men } \\
\text { and women aged } 20- \\
50 \text { years }\end{array}$ & $\begin{array}{l}\text { Pranayama } \\
\text { for } 6 \text { weeks }\end{array}$ & $\begin{array}{l}\text { No practice at } \\
\text { all }\end{array}$ & $\begin{array}{l}\text { Systolic blood } \\
\text { pressure and } \\
\text { heart rate }\end{array}$ \\
\hline $\begin{array}{l}\text { Kalavani } \\
\text { (2019) }\end{array}$ & India & $\begin{array}{l}\text { Patients with grade } 1 \\
\text { hypertension and } \\
\text { prehypertension, } \\
\text { men and women } \\
\text { aged } 30-60 \text { years }\end{array}$ & $\begin{array}{l}\text { Alternate } \\
\text { Nostil } \\
\text { Breathing } \\
10 \text { minutes/ } \\
\text { session, for } \\
5 \text { days }\end{array}$ & $\begin{array}{l}\text { No practice at } \\
\text { all }\end{array}$ & $\begin{array}{l}\text { Systolic blood } \\
\text { pressure, } \\
\text { diastolic, heart } \\
\text { rate, }\end{array}$ \\
\hline $\begin{array}{l}\text { Misra } \\
(2019)\end{array}$ & Columbia & $\begin{array}{l}\text { Patients with grade } 1 \\
\text { and } 2 \text { hypertension, } \\
\text { men and women } \\
\text { aged } 60 \text { years }\end{array}$ & $\begin{array}{l}\text { Pranayama } \\
15 \text { minutes/ } \\
\text { session, } 5 \\
\text { times a } \\
\text { week for } 6 \\
\text { weeks }\end{array}$ & $\begin{array}{l}\text { No practice at } \\
\text { all }\end{array}$ & $\begin{array}{l}\text { Systolic blood } \\
\text { pressure }\end{array}$ \\
\hline $\begin{array}{l}\text { Sathe } \\
(2020)\end{array}$ & India & $\begin{array}{l}\text { Hypertensive } \\
\text { patients, male and } \\
\text { female aged } 40 \text { years }\end{array}$ & $\begin{array}{l}\text { Bhramari } \\
\text { Pranayama } \\
5 \text { minutes/ } \\
\text { session }\end{array}$ & $\begin{array}{l}\text { No practice at } \\
\text { all }\end{array}$ & $\begin{array}{l}\text { Systolic blood } \\
\text { pressure, } \\
\text { diastolic, heart } \\
\text { rate, } \mathrm{SpO} 2\end{array}$ \\
\hline $\begin{array}{l}\text { Shetty } \\
(2017)\end{array}$ & India & $\begin{array}{l}\text { Patients with grade } 1 \\
\text { hypertension and } \\
\text { prehypertension, } \\
\text { men and women } \\
\text { aged } 25-65 \text { years }\end{array}$ & $\begin{array}{l}\text { Sheetali \& } \\
\text { Sheetkari } \\
\text { Pranayama } \\
10 \text { min/ } \\
\text { session }\end{array}$ & $\begin{array}{l}\text { No practice at } \\
\text { all }\end{array}$ & $\begin{array}{l}\text { Systolic blood } \\
\text { pressure, heart } \\
\text { rate, } \\
\text { respiratory rate }\end{array}$ \\
\hline $\begin{array}{l}\text { Telles } \\
(2013)\end{array}$ & India & $\begin{array}{l}\text { Grade } 1 \text { essential } \\
\text { hypertension } \\
\text { patients, men and } \\
\text { women aged 20-59 } \\
\text { years }\end{array}$ & $\begin{array}{l}\text { Alternate } \\
\text { Nostil } \\
\text { Breathing } \\
\text { 10 minutes/ } \\
\text { session }\end{array}$ & $\begin{array}{l}\text { No practice at } \\
\text { all }\end{array}$ & $\begin{array}{l}\text { Systolic and } \\
\text { diastolic blood } \\
\text { pressure }\end{array}$ \\
\hline $\begin{array}{l}\text { Thanalaks } \\
\text { hmi } \\
\text { (2020) }\end{array}$ & India & $\begin{array}{l}\text { Essential } \\
\text { hypertension } \\
\text { patients, men and } \\
\text { women aged 18-6o } \\
\text { years }\end{array}$ & $\begin{array}{l}\text { Sheetali } \\
\text { Pranayama } \\
\text { 30 } \\
\text { minutes/ } \\
\text { session for } \\
4 \text { weeks }\end{array}$ & $\begin{array}{l}\text { No practice at } \\
\text { all }\end{array}$ & $\begin{array}{l}\text { Systolic and } \\
\text { diastolic blood } \\
\text { pressure }\end{array}$ \\
\hline
\end{tabular}


Khairunissa et al./ Aerobic Exercise and Yoga Breathing Exercise (Pranayama) on Blood Pressure

\begin{tabular}{|c|c|c|c|c|c|c|c|c|c|c|c|c|}
\hline \multirow{3}{*}{$\begin{array}{l}\text { Study or Subgroup } \\
\text { Dixit } 2017\end{array}$} & \multicolumn{3}{|c|}{ pranayama } & \multicolumn{3}{|c|}{ no pranayama } & \multicolumn{2}{|r|}{ Std. Mean Difference } & \multirow{2}{*}{\multicolumn{4}{|c|}{$\begin{array}{l}\text { Std. Mean Difference } \\
\text { IV, Random, } 95 \% \mathrm{Cl}\end{array}$}} \\
\hline & Mean & SD & Total & Mean & SD & Total & Weight & IV, Random, $95 \% \mathrm{Cl}$ & & & & \\
\hline & 129.05 & 3.86 & 17 & 144.87 & 2.3 & 17 & $9.1 \%$ & $-4.86[-6.26,-3.46]$ & & & & \\
\hline Ghati 2020 & 131.7 & 10.9 & 32 & 125.8 & 12.6 & 35 & $11.7 \%$ & $0.49[0.01,0.98]$ & & & - & \\
\hline Goyal 2014 & 127 & 2.42 & 25 & 141 & 2.41 & 25 & $9.5 \%$ & $-5.71[-7.00,-4.42]$ & & $\longrightarrow$ & & \\
\hline Kalavani 2019 & 80.42 & 2.897 & 85 & 84.49 & 4.492 & 85 & $12.0 \%$ & $-1.07[-1.39,-0.75]$ & & $=$ & & \\
\hline Misra 2018 & 152.1 & 19.1 & 23 & 151.6 & 18.9 & 23 & $11.6 \%$ & $0.03[-0.55,0.60]$ & & & & \\
\hline Sathe 2020 & 118.85 & 9.83 & 20 & 127.28 & 8.82 & 20 & $11.4 \%$ & $-0.88[-1.54,-0.23]$ & & $\rightarrow$ & & \\
\hline Shetty 2017 & 132 & 3.8 & 30 & 152.9 & 9.8 & 30 & $11.2 \%$ & $-2.78[-3.50,-2.05]$ & & $\rightarrow$ & & \\
\hline Telles 2013 & 128 & 10.64 & 31 & 139.53 & 18.65 & 31 & $11.7 \%$ & $-0.75[-1.27,-0.23]$ & & $\rightarrow$ & & \\
\hline Thanalaksami 2020 & 124.12 & 14.72 & 40 & 139.72 & 12.9 & 42 & $11.8 \%$ & $-1.12[-1.59,-0.65]$ & & $\rightarrow$ & & \\
\hline Total $(95 \% \mathrm{Cl})$ & & & 303 & & & 308 & $100.0 \%$ & $-1.68[-2.52,-0.84]$ & & & & \\
\hline \multicolumn{9}{|c|}{$\begin{array}{l}\text { Heterogeneity: } \text { Tau }^{2}=1.50 ; \mathrm{Ch}^{2}=153.09, \mathrm{df}=8(\mathrm{P}<0.00001) ; \mathrm{I}^{2}=95 \% \\
\text { Test for overall effect: } Z=3.93(\mathrm{P}<0.0001)\end{array}$} & -10 & $\begin{array}{l}-5 \\
\text { pranayama }\end{array}$ & no pranay & $\begin{array}{l} \\
5 \\
\text { yama }\end{array}$ \\
\hline
\end{tabular}

\section{Figure 6. Forest plot of the effect of yoga breathing exercise (pranayama) on systolic blood pressure in hypertensive patients}

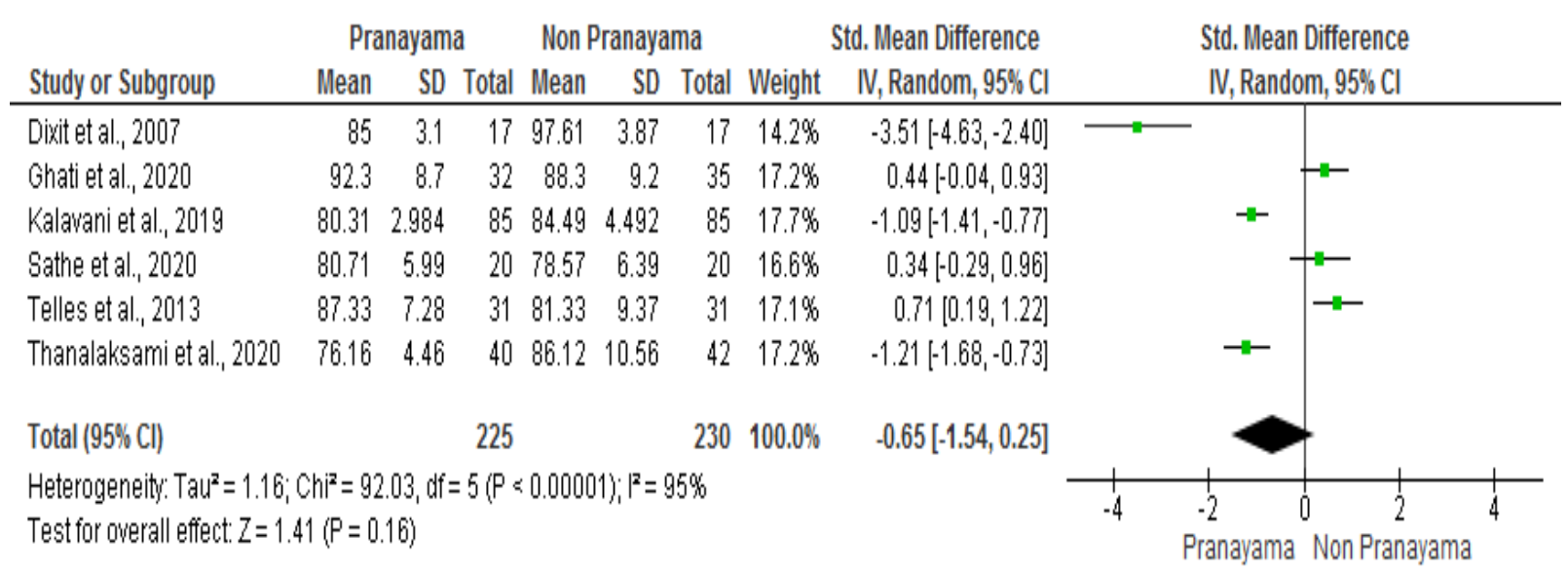

\section{Figure 7. Forest plot of the effect of aerobic exercise on diastolic blood pressure in hypertensive patients}

\section{b. Funnel plot}

In Figure 8, the funnel plot graph shows an asymmetrical distribution of the primary study, 3 plots on the left and 6 plots on the right, indicating that there is a slight publication bias that tends to overestimate the true effect of yoga breathing exercise (pranayama) interventions on reducing systolic blood pressure (overestimated). ).
In Figure 9, the funnel plot graph shows the asymmetric distribution of the primary study. The funnel plot indicates that there is a slight publication bias that tends to overestimate the true effect of the yoga breathing exercise (pranayama) intervention on reducing diastolic blood pressure (overestimate). 
Khairunissa et al./ Aerobic Exercise and Yoga Breathing Exercise (Pranayama) on Blood Pressure

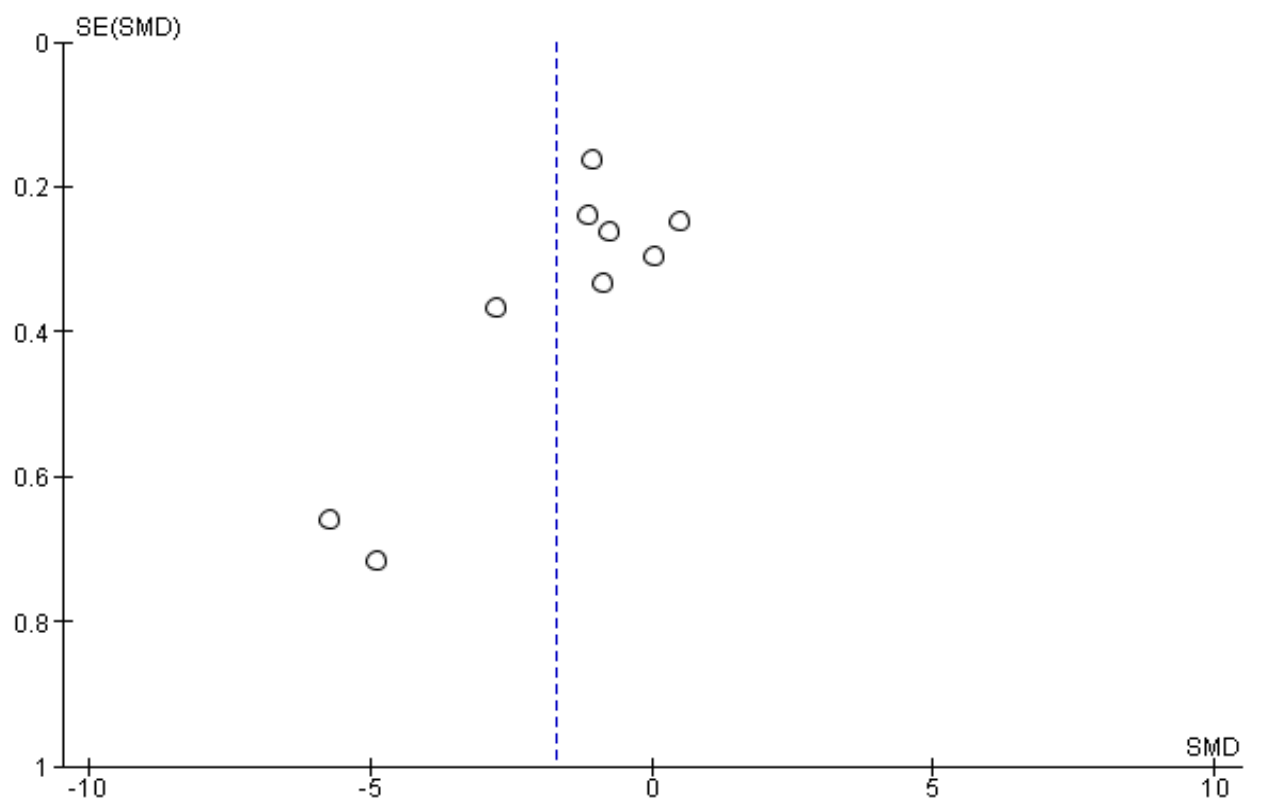

Figure 8. Funnel plot of the effect of yoga breathing exercise (pranayama) on systolic blood pressure in hypertensive patients

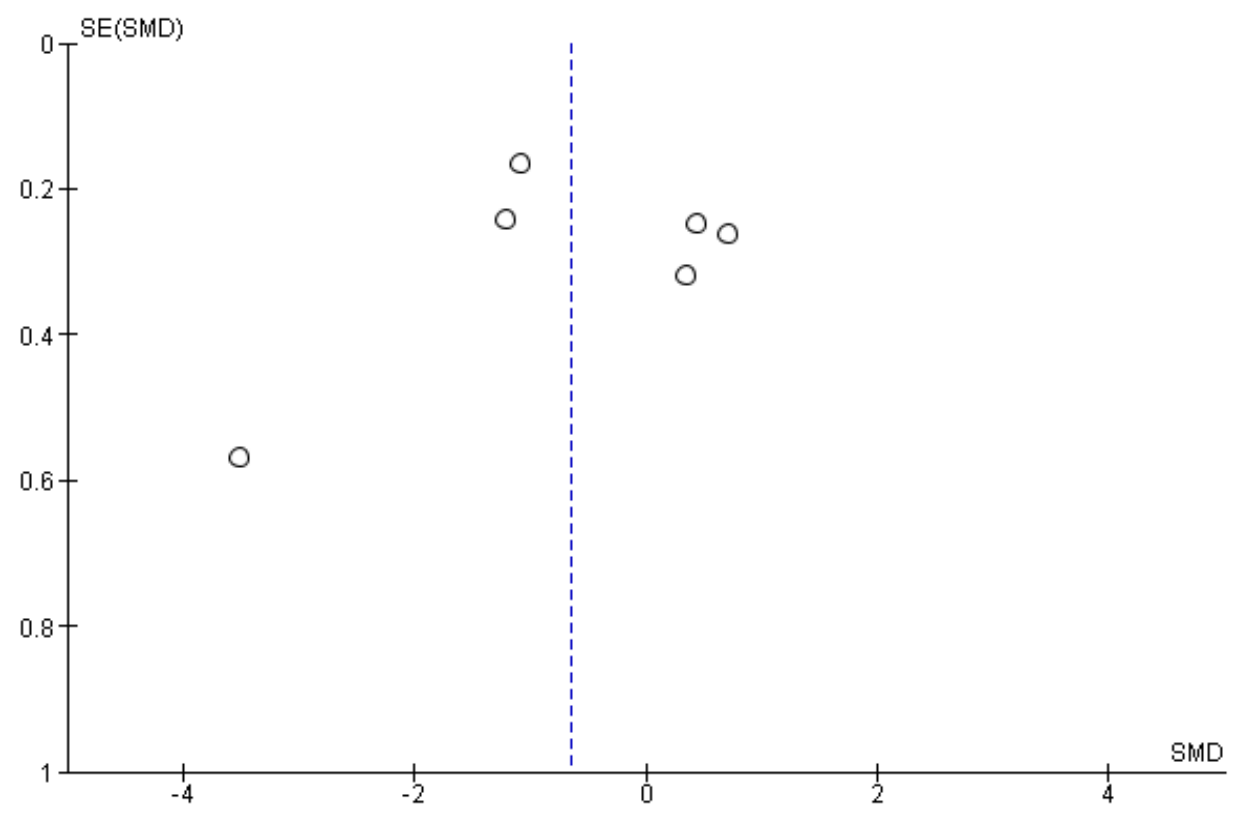

Figure 9. Funnel plot of the effect of yoga breathing exercise (pranayama) on diastolic blood pressure in hypertensive patients

\section{DISCUSSION}

Hypertension is a cardiovascular risk factor that causes an increase in morbidity and mortality (Osuji et al., 2012). The use of antihypertensive drugs has been shown to provide effective lowering of blood pressure, but some antihypertensive drugs affect lipoprotein metabolism which can increase the risk of cardiovascular disease, thereby closing the benefits in lowering blood pressure (Deshmukh et al., 2008). 
The potential side effects of some antihypertensive drugs on lipoprotein metabolism does not mean that the therapy should be discontinued, but that other complementary therapies are needed to strengthen the benefit of therapy in lowering blood pressure. The American, Canadian, and European hypertension guidelines recommend non-pharmacological interventions in the prevention and management of hypertension (Williams et al., 2018) (Whelton et al., 2018) (Leung et al., 2017). Non-pharmacological intervenetions help reduce the daily dose of antihypertensive drugs and delay the development of hypertension, as well as reduce blood pressure in hypertensive patients. These interventions emphasize the importance of lifestyle interventions such as physical exercise and yoga (Mahmood et al., 2018). The recommended form of physical exercise is aerobic exercise and part of yoga practice in the form of breathing exercises or commonly called yoga breathing exercise (pranayama) has a beneficial impact in reducing blood pressure (Unger et al., 2020).

This study uses a systematic review and meta-analysis study design with the aim of obtaining general conclusions as the basis for providing therapy/intervention from various similar studies that have been carried out by previous researchers who tested the provision of aerobic exercise and yoga breathing exercise (pranayama) in hypertensive patients in various countries. country.

Meta-analysis is an epidemiological study that combines and statistically combines data from primary research results that discuss the same hypothesis so as to obtain quantitative summary results (Egger and Smith in Murti, 2018). The results of the research are presented in the form of forest plots and funnel plots.
Forest plots can show effect sizes and 95\% confidence intervals or display results from meta-analysis studies (Akobeng, 2005 in Murti, 2018). The funnel plot shows the effect size and precision of the effect size and makes it possible to evaluate the possibility of publication bias in the form of a symmetrical triangle graphic (Murti, 2018).).

\section{Aerobic exercise on blood pressure}

Giving aerobic exercise to hypertensive patients was able to significantly reduce systolic blood pressure by 0.93 times and diastolic blood pressure by 0.56 compared to non-aerobic exercise interventions. The results of this study are supported by research by Daimo et al., (2020), where giving moderate intensity aerobic exercise (60\% HR) in the form of brisk walking for 16 weeks can significantly reduce systolic and diastolic blood pressure compared to the control group. Likewise with Lamina's research (2010) which suggests giving aerobic exercise to reduce blood pressure in hypertensive patients as an effort to reduce the incidence of cardiovascular disease.

Giving pure aerobic exercise has shown the natural benefits of lowering blood pressure, but based on the research of Sousa et al., (2013) stated that aerobic exercise will provide a greater reduction in blood pressure when combined with resistance exercise. This is due to another effect resulting from the combination of exercise, namely a reduction in the percentage of body fat which is correlated with changes in blood pressure. In line with this, Garber et al., (2011) in their research recommend aerobic exercise with moderate to strong intensity with a duration of 3 times a week and it would be better if added with resistance exercise 2 or more times a week.

Basically, aerobic exercise which has rules ranging from frequency, intensity, time, and type is an alternative physical 
exercise that can be done by hypertensive patients and is expected to be part of a lifestyle and an effort to control hypertension (Pescatello et al., 2015).

\section{Yoga breathing exercise on blood pressure}

Giving yoga breathing exercise (pranayama) to hypertensive patients was able to significantly reduce systolic blood pressure by 1.68 times than non-yoga breathing exercise (pranayama) intervention. Unlike the diastolic blood pressure which decreased 0.65 times than the non-yoga breathing exercise intervention (pranayama) but not statistically significant. In the study of Ghati et al., (2020) also wrote that giving bee humming pranayama can reduce blood pressure even though it is not statistically significant.

Some of the factors that caused the results of an insignificant decrease in blood pressure including first, the research subjects had taken antihypertensive drugs so that they had controlled blood pressure before exercise, Second, the condition of the sample did not know the pranayama technique and was only doing it for the first time causing a possible discrepancy in practice. Third, the short duration of the practice of 5 minutes in 5 days, if the duration was extended it might yield more explanations regarding the effectiveness of pranayama primarily on blood pressure. Fourth, this study involved a small sample while the predictive power would be better if the sample size was larger. In line with this, Sathe et al., (2020) reported that bharamari pranayama given to 20 subjects for 5 days had an insignificant effect in reducing systolic and diastolic blood pressure in hypertensive patients.

However, there is a difference in the study of Thanalakshmi et al., (2020) which states that the administration of sheetali pranayama intervention 20 repetitions for
4 weeks can reduce systolic blood pressure 1.04 times and lower diastolic blood pressure $\mathbf{1 . 0 2}$ times compared to the control group and is statistically significant $(p<0.001)$. Based on this, the duration and technique of the exercise must be considered in the pranayama intervention in order to produce the maximum effect.

The limitations of this study are publication bias, search bias and language bias. It is hoped that further research can conduct research using a larger number of subjects and a higher timeframe and follow-up.

\section{AUTHOR CONTRIBUTION}

Khairunissa is the main researcher who selects the topic, searches and collects research data. Didik Tamtomo and Hanung Prasetya analyzed data and reviewed research documents.

\section{FUNDING AND SPONSORSHIP}

This study is self-funded.

\section{CONFLICT OF INTEREST}

There is no conflict of interest in this study.

\section{ACKNOWLEDGEMENT}

We are very grateful to database providers PubMed, Science Direct, Research Gate, and Google Scholars.

$\begin{array}{ll} & \text { LIST OF ABBREVIATIONS } \\ \mathrm{CAD} & =\text { Coronary Artery Disease } \\ \mathrm{CI} & =\text { Confident Interval } \\ \mathrm{CKD} & =\text { Chronic Kidney Disease } \\ \mathrm{HF} & =\text { Heart Failure } \\ \mathrm{PICO} & =\text { Population, Intervention } \\ & \text { Comparisons, Outcome } \\ \mathrm{RCT} & =\text { Randomized Controlled Trial } \\ \mathrm{SMD} & =\text { Standard Mean Difference } \\ \mathrm{WHO}= & \text { World Health Organization }\end{array}$




\section{REFERENCES}

Aflahiyah S, Tamtono DG, Prasetya H (2020). Effectiveness of prenatal yoga on sleep quality and reduction of anxiety during pregnancy: A metaanalysis. J Matern Child Health. 5(6): 629-640. https://doi.org/10.26911/thejmch.2020.05.06.03

Astuti EP, Suwardianto H, Yuliantin N (2016). 5 th octave music therapy is decrease blood pressure to patients with hypertension. Jurnal Penelitian Keperawatan. 2(1). https://doi.org/10.3266o/jurnal.v2i1.146

Armstrong N, Mc Manus A (eds). (2017). Oxford Texbook of Children's Sport and Exercise Medicine. Third. doi: 10.1007/978-1-4612-3182-0_2.

Daimo M, Mandal S, Abdulkader M, Mathivanan D (2020). Effect of aerobic exercise on blood pressure in men with hypertension: A randomized controlled study. Turk J Kinesiol. 6(1): 32-39. doi: 10.31459/turkjkin.686578.

Deshmukh M, Lee Ho Won, McFarlane, Samy I, Whaley C, Adam (2008). Antihypertensive medications and their effects on lipid metabolism. Current Diabetes Reports. 8(3): 214-220. doi: 10.1007/s11892-008-0037-7.

Dixit R, Ranjan ST, Dixit M (2017). An evaluation of the effect of Pranayamas and Transcendental meditation on high blood pressure subjects: A nonblinded randomized controlled trial. IJCAP. 4(2): 254. doi: 10.18231/23942126.2017.0063.

Dorobantu M, Mancia G, Grassi G, Voicu V (eds). (2019). Hypertension and Heart Failure: Epidemiology, Mechanisms and Treatment, Heart Failure Clinics. Switzerland: Springer Nature Switzerland. doi: 10.1016/j.hfc.2019.06.007.

Fu J, Liu Y, Zhang L, Zhou L, Li D, Quan H,
Zhu L, et al. (2020). Nonpharmacologic interventions for reducing blood pressure in adults with prehypertension to established hypertension. J Am Heart Assoc. 9(19): e016804. https://doi.org/10.1161/jaha.120.016 804.

Garber CE, Blissmer, Bryan D, Michael RF, Barry AL, Michael JL, I Min N, et al. (2011). Quantity and quality of exercise for developing and maintaining cardiorespiratory, musculoskeletal, and neuromotor fitness in apparently healthy adults: Guidance for prescribing exercise. Med Sci Sports Exerc. 43(7): 1334-59. https://doi.org/10.1249/mss.obo13e318213fefb.

Ghati N, Sharma G, Karunakaran B, Agarwal A, Mohanty S, Nivethitha L, Siddharthan D, et al. (2020). A randomized trial of the immediate effect of Bee-humming breathing exercise on blood pressure and heart rate variability in patients with essential hypertension. Explore (NY). 17(4): 312-319. https://doi.org/10.1016/j.explore.2020.03.009.

Lamina S (2010). Effects of continuous and interval training programs in the management of hypertension: A randomized controlled trial. J Clin Hypertens. 12(11): 841-849. https://doi.org/10.1111/j.1751-7176.2010.00315. $x$.

Leung AA, Daskalopoulou SS, Dasgupta K, McBrien K, Butalia S, Zarnke KB, Nerenberg K, et al. (2017). Hypertension Canada's 2017 guidelines for diagnosis, risk assessment, prevention, and treatment of hypertension in adults. Can J Cardiol. 33(5): 557-576. https://doi.org/10.1016/j.cjca.2017.o 3.005 .

Lindasari N, Suhariyanti E, Margowati S (2020). Yoga Pranayama Dhiirga 
Khairunissa et al./ Aerobic Exercise and Yoga Breathing Exercise (Pranayama) on Blood Pressure

Swasam with Sukhasana Position on reducing blood pressure in the elderly. Atlantis Press. 333-338. https://dx.doi.org/10.2991/assehr.k.200529. 068.

Mahmood S, Shah KU, Khan TM, Nawaz S, Rashid H, Baqar SWAB, Kamran S (2018). Non-pharmacological management of hypertension: in the light of current research. Ir J Med Sci. 188(2): 437-452. https://doi.org/10.1007/s11845-018-1889-8.

Murti B (2018). Prinsip dan metode riset epidemiologi (Principles and methods of epidemiological research). V. Karanganyar: Program Studi Ilmu Kesehatan Masyarakat.

Ningrum SA, Budihastuti UR, Prastya $H$ (2019). Efficacy of yoga exercise to reduce anxiety in pregnancy: A metaanalysis using randomized controlled trials. J Matern Child Health. 4(2): 118-125. https://doi.org/10.26911/thejmch.2019.04.02.07

Osuji CU, Omejua EG, Onwubuya EI, Ahaneku GI (2012). Serum lipid profile of newly diagnosed hypertensive patients in Nnewi, South-East Nigeria. Int J Hypertens. 2012:710486. https://doi.org/10.1155/2012/710486.

Pescatello LS, MacDonald HV, Lamberti L, Johnson BT (2015). Exercise for hypertension: A prescription update integrating existing recommendations with emerging research. (17): 87. doi: 10.1007/s11906-015-0600-y.

Sathe S, Thodge K, Rajandekar T, Agrawal A (2020). To find out immediate effect of bhramari pranayama on blood pressure, heart rate and oxygen saturation in hypertensive patients. Int J Curr Res Rev. 12(19): 193-197. https://doi.org/10.31782/IJCRR.202 0.121919 .

Unger T, Borghi C, Charchar F, Nadia AK, Poulter NR, Prabhakaran D, Ramirez A, et al. (2020). 2020 International society of hypertension global hypertension practice guidelines. AHA. 75(6). doi: 10.1161/HYPERTENSIONAHA.120.15026.

Weber MA, Schiffrin EL, White WB, Mann S, Lindholm LH, Kenerson JG, Flack JM, Carter BL, et al. (2014). Clinical practice guidelines for the management of hypertension in the community: A statement by the american society of hypertension and the international society of hypertension. J Clin Hypertens. 16(1): 14-26. doi: 10.1111/jch.12237.

Whelton PK, Carey RM, Aronow WS, Casey DE, Collins KJ, Himmelfarb CD, De Palma SM, et al. (2018). 2017 ACC/AHA/AAPA/ABC/ACPM/AGS/ APhA/ASH/ASPC/NMA/PCNA guideline for the prevention, detection, evaluation, and management of high blood pressure in adults. ACC/AHA. doi: 10.1161/HYP.o000000000000065.

Williams B, Mancia G, Spiering W, Rosei E A, Burnier M, Clement DL, Coca A, et al. (2018). 2018 ESC/ESH Guidelines for the Management of Artial Hypertension. EHJ. 1-98. doi: 10.1097/HJH.0ooooooooooo1961. 